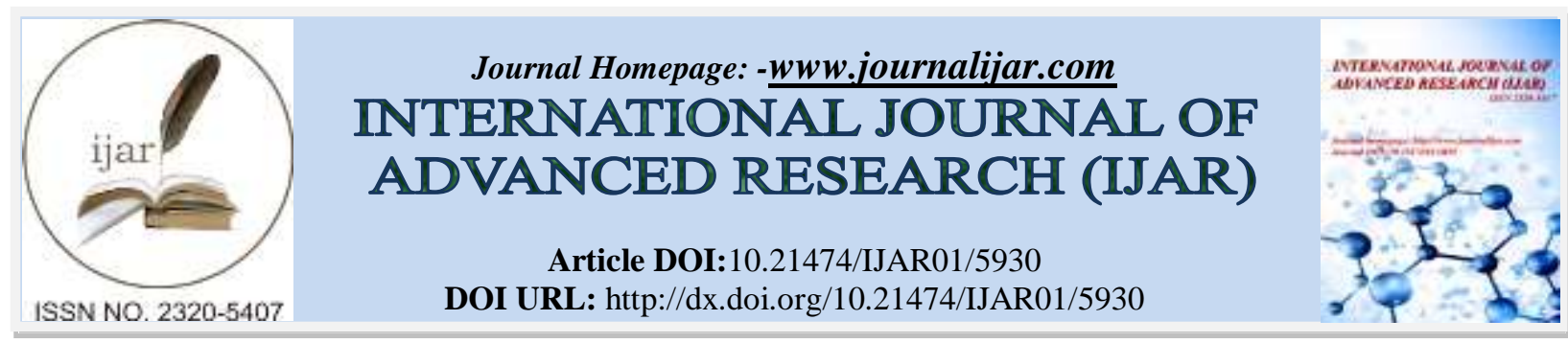

RESEARCH ARTICLE

\title{
EVALUATION OF MALOCCLUSION BY USING PANORAMIC RADIOGRAPHS.
}

\author{
Dr. Sanket Mendhe ${ }^{1}$, Dr. Tushar Manohar Rothe ${ }^{2}$, Dr. PriteshGangde ${ }^{3}$, Dr. AroraAbhas ${ }^{4}$, Dr. \\ AddityaMarkandey ${ }^{5}$ and Dr. MohdTabish Rashidi. \\ 1. Department of Orthodontics, Dr. Rajesh Kambe Dental College and Hospital, Akola, India. \\ 2. Department of Oral and Maxillofacial Surgery, Dr. Rajesh Kambe Dental College and Hospital, Akola, India. \\ 3. Department of Prosthodontics, Dr. Rajesh Kambe Dental College and Hospital, Akola, India. \\ 4. Department of Public Health Dentistry, Dr. Rajesh Kambe Dental College and Hospital, Akola, India. \\ 5. Department of Oral and Maxillofacial Surgery, Dr. Rajesh Kambe Dental College and Hospital, Akola, India. \\ 6. Department of Prosthodontics, People's College of dental science and research centre.
}

\section{Manuscript Info}

Manuscript History

Received: 01 October 2017

Final Accepted: 03 November 2017

Published: December 2017

Key words:-

OPG, Malocclusion, lateral cephalogram.

\section{Abstract}

Orthodontics is a specialized branch dealing with the assessment and treatment of malocclusion. This discrepancy in dento-skeletal structure may be due to disproportionate sizes of jaws or tooth material and arch length deficiency or presence of both. To assess the presence of malocclusion various modalities can be use which includes clinical examination, case history, photographs, study models and various type of radiographs like IOPA, OPG, and lateral cephalograms. Radiology has long played a critical role in the treatment planning of orthodontic patients. Since the discovery of lateral cephalogram, it has played a distinct role to assess the craniofacial growth and presence of malocclusion. This study included 60 patients out of which 30 males and 30 females between the age group of 18-25 years. The patients with malocclusion after the completion of growth were included in this study. Patients with missing any first molar, periodontaly compromised tooth, restorated tooth or any history of orthodontic treatment were excluded in this study. Tracing was done on acetate paper using a 0.5 $\mathrm{mm}$ lead pencil. Angular measurements were made to 0.5accuracy. Measurements on panoramic radiographs were made for both the left and right sides to overcome any magnification errors.

Copy Right, IJAR, 2017,. All rights reserved.

\section{Introduction:-}

Orthodontics is a specialized branch dealing with the assessment and treatment of malocclusion. This discrepancy in dentoskeletal structure may be due to disproportionate sizes of jaws or tooth material and arch length deficiency or presence of both. To assess the presence of malocclusion various modalities can be use which includes clinical examination, case history, photographs, study models and various type of radiographs like IOPA, OPG, and lateral cephalogram. Radiology has long played a critical role in the treatment planning of orthodontic patients. Since the discovery of lateral cephalogram, it has played a distinct role to assess the craniofacial growth and presence of malocclusion. Cephalometric analysis has been considered to be the gold standard for diagnosis and treatment planning at the start of orthodontic treatment. Assessment of craniofacial skeleton is frequently compromised by the variability existing both between and within individuals. Though lateral cephalogram provides lot of information 
regarding the craniofacial skeleton, it is impossible to visualize accurately the right and left sides of these structures in a single radiograph due to superimposition of two sides. Other limitations include radiographic film magnification, tracing, measuring, recording and identifying the landmarks.[3]

Panoramic radiograph was developed by Paatero YV in 1948, It allows the visualization of both the right and left side of craniofacial structures by producing an accurate, predictable image of all the teeth and related structures on radiograph, in shortest possible time, with least amount of radiation exposure to the patient and to the operator and with the minimal amount of superimposition of various structures.[4,5] Panoramic radiography is frequently used in orthodontic practice to provide important information about the teeth and structure of bony architecture supporting the surrounding teeth, their axial inclinations, maturation periods and surrounding tissues [1]. Panoramic radiograph is usually the technique of choice because of its relatively low radiation exposure, degree of patient comfort and the significant amount of diagnostic information obtained by viewing all the teeth and basal bone [2]. The advantages of a panoramic radiography are that it permits visualization of both the maxilla and mandible on one film. The film permits a determination of the number of teeth present (with some caution), their positions and evaluation of gross osseous changes in the condyles and is quite helpful in diagnosis of cases. The relation between structures is accurately depicted so that the clinician may compare the relationship of teeth with one another and to other structures. 3 therefore, panoramic radiography has become an indispensable orthodontic screening tool.

In present study we can determine the potential of panoramic radiography for evaluating craniofacial specifications and angulation of axial inclination of teeth as compared to lateral cephalograms by evaluating various skeletal and dental parameters.

\section{Materials And Methods:-}

All the procedure was done by single operator to avoid errors due to inter operator variations. The study was conducted in the Department of Orthodontics and DentofacialOrthopaedics. The study included 60 patients out of which 30 males and 30 females between the age group of 18-25 years. The patients with malocclusion after the completion of growth were included in this study. Patients with missing any first molar, periodontaly compromised tooth, restorated tooth or any history of orthodontic treatment were excluded in this study.

Panoramic radiographs and lateral cephalograms of the 60 patients includes 30 males and 30 females were selected according to the above mentioned criteria. The radiographs were made in standardized conditions with clinical Frankfort horizontal plane (FHP) kept parallel to the floor and the mid facial plane kept in a vertical position using the Advanced graphics systems ( $80 \mathrm{kv} \max /$ total filtration $2.5 \mathrm{~mm} \mathrm{AL}$ )

Tracing was done on acetate paper using a $0.5 \mathrm{~mm}$ lead pencil. Angular measurements were made to 0.5 accuracy. Measurements on panoramic radiographs were made for both the left and right sides to overcome any magnification errors.

The bite plate used in the panoramic radiographs for altered occlusion. Therefore independent reference planes were set up in the maxilla and the mandible on the panoramic images. FHP was constructed between meatus acusticusexternus (Mae) and orbital points. A reference plane was drawn between the intersection point of the ascending and descending tangents on the Mandibular canal (MC) and foramen Mentale (FME). The bite plate caused the mandible to shift forward. Materials and Methods

\begin{tabular}{|l|l|}
\hline $\begin{array}{l}\text { The following panoramic landmarks were } \\
\text { indentified. }\end{array}$ & \\
\hline Landmarks & Significance \\
\hline S & Sella: geometric center of pituitary fossa \\
\hline N & Nasion : most anterior aspect of the frontonasal suture \\
\hline Or & Orbitale: lowest point on the inferior rim of the orbit \\
\hline ANS & $\begin{array}{l}\text { Anterior nasal spine: anterior tip of the sharp bony } \\
\text { process of the maxilla at the lower margin of the anterior } \\
\text { nasal opening }\end{array}$ \\
\hline PNS & $\begin{array}{l}\text { Posterior nasal spine: posterior spine of the palatine } \\
\text { bone constituting the hard palate }\end{array}$ \\
\hline A-POINT & Subspinale: most posterior midline point on the \\
\hline
\end{tabular}




\begin{tabular}{|l|l|}
\hline & $\begin{array}{l}\text { concavity between anterior nasal spine and the } \\
\text { prosthion. }\end{array}$ \\
\hline U1 & Incisal edge of maxillary incisor \\
\hline L1 & Incisal edge of mandibular incisor \\
\hline U6 & Distobuccaltubercule of maxillary first molar \\
\hline Gn & Distobuccaltubercule of mandibular first molar \\
\hline Me & $\begin{array}{l}\text { Gnathion : midpoint between Pogonion and Menton } \\
\text { mantion : lowest point on the symphysis shadow of the }\end{array}$ \\
\hline Go & $\begin{array}{l}\text { Gonion: a point on the curvature of the angle of } \\
\text { mandible located by bisecting the angle formed by line } \\
\text { tangent to the posterior ramus and inferior border of the } \\
\text { mandible. }\end{array}$ \\
\hline Mae & $\begin{array}{l}\text { Meatus acusticusexternus: external auditory meatus } \\
\text { condylion: posterior superior point on the head of the }\end{array}$ \\
\hline Co & \\
\hline
\end{tabular}

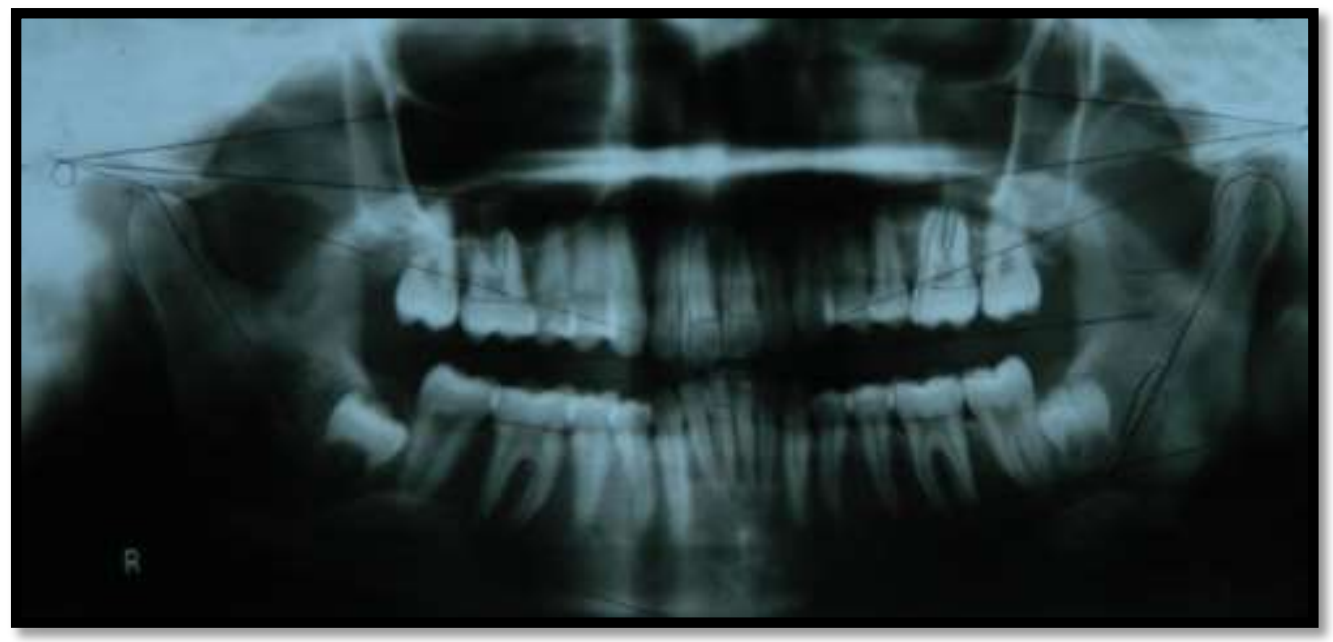

photograph no. 5:-Tracing On Orthopantomogram

\begin{tabular}{|c|c|c|}
\hline $\begin{array}{c}\text { Angles used for assessment } \\
\text { of- }\end{array}$ & Landmarks used in cephalogram & $\begin{array}{c}\text { Landmarks used in panoramic } \\
\text { Radiograph }\end{array}$ \\
\hline $\begin{array}{c}\text { Skeletal antero posterior } \\
\text { dysplasia of the maxilla }\end{array}$ & Sella-Nasion to point A & Orbitale-Porion-ANS \\
\hline Morphology of the mandible & CondylionGonionMenton & CondylionGonionMenton \\
\hline Cant of occlusal plane & $\begin{array}{c}\text { Intersection of Occlusal plane and } \\
\text { Pori on Orbital plane }\end{array}$ & $\begin{array}{c}\text { Intersection of Occlusal plane and } \\
\text { PorionOrbitale plane }\end{array}$ \\
\hline Regress Equation & Go - Gn - SN & OMAND \\
\hline
\end{tabular}

The angular measurements measured on the lateral cephalogram and compared on the panoramic radiograph. ${ }^{4}$

\section{Results:-}

Lateral cephalograms and Orthopantomograms were taken of a total of 60 patients includes 30 males and 30 females with skeletal malocclusion and dental malocclusion. These were recorded with the objective of evaluating and comparing craniofacial characteristics, and to investigate the possibility of enhancing the clinical versatility of the panoramic radiographs.

On measuring each parameter on the orthopantomogram the following findings were noted. 


\section{FH/ANS (Relation of Frankfort's horizontal to anterior nasal spine):-}

On comparison of males and females no statistically significant difference was found within the sample (Table IV). On comparison of the right and left sides a mean difference of 1.450 and standard deviation of 1.71163 was noted which was statistically found to be very highly significant $(0.001)$ [Table V]. Correlation with cephalometric relation of palatal plane to mandibular plane was found to be (.081) and relation of cranial base to mandibular plane was (.206). It showed a negative correlation to the cephalometricgonial angle (-.115) and condylar inclination angle (.150) $[$ Table VI].

OMAND (Panoramic alternative of cephalometricgonial angle):-

On comparison between males and females no statistically significant difference was noted within the sample [Table IV]. On comparison of the right and left sides a mean of .5833 and standard deviation of 4.11402 was calculated which was found to be statistically insignificant [Table V]. On correlation with cephalometric readings it was found that OMAND showed a positive correlation with cephalometric condylar inclination angle (.318), relation of mandibular plane to cranial base (.210) and relation between palatal plane and mandibular plane (.165). Therefore OMAND could be used as a panoramic alternative to measure the cephalometricgonial angle using the following regression equation i.e. $\mathrm{Co}-\mathrm{Go} / \mathrm{Go}-\mathrm{Me}=72.864( \pm 19.425)+[0.367( \pm .144)]$ OMAND. This showed a prediction value of $10.1 \%$.

FH/UOP (Angle between Frankfort's horizontal and maxillary occlusal plane):-

On comparison between males and females no statistically significant difference was found within the sample [Table IV]. On comparison between the right and the left sides a mean of .150 and standard deviation of 2.64142 was calculated which was found to be statistically insignificant [Table V]. On correlation with cephalometric readings it was found that FH/UOP showed a positive correlation with cephalometric angle between Frankfort's horizontal and maxillary occlusal planes (.125) [Table VII]. On correlation with panoramic angle between Frankfort's horizontal and mandibular occlusal plane it showed a positive correlation (.415) [Table VIII]. Maxillary occlusal plane there was subsequent increase in the mandibular occlusal plane.

FH/LOP (Angle between Frankfort's horizontal and mandibular occlusal planes):-

On comparison between males and females no statistically significant difference was noted within the sample. On comparison between the right and left side a mean of 1.667 with standard deviation of 2.40527 was measured which was found to be statistically very highly significant (0.001). On correlation with cephalometric values FH/LOP showed a positive correlation with angle between Frankfort's horizontal and lower occlusal plane (.270).

\section{OCOND (Panoramic radiograph alternative of condylar inclination angle):-}

On Comparison of males and females no statistically significant difference was noted within the sample. On comparison between the right and the left sides a mean of 1.3833 and standard deviation of 6.12605 was measured which was found to be statistically insignificant [Table V]. On correlation with cephalometric values it showed a negative correlation with condylar inclination angle (-.305), palatal plane to mandibular plane (-.279) and relation of cranial base to mandibular plane (-.397). Therefore OCOND can be used as a panoramic alternative to determine cephalometric relation of cranial base to mandibular plane and palatal plane to mandibular plane with the following regression equations respectively.

Go-Gn/S-N=49.711 ( \pm 7.126$)-[0.46( \pm .139)]$ OCOND

ANS-PNS/Go-Me=34.278( \pm 5.829$)-[0.253( \pm .114)] \mathrm{OCOND}$

OMID (angle between Frankfort's horizontal and maxillary incisors):-

On comparison between males and females no statistically significant difference was found within the sample. On comparison between right and left sides a mean of 1.8667 and standard deviation of 2.38261 was calculated which was found to be statistically very highly significant. On correlation with cephalometric parameters it showed a positive correlation (.458).

\section{Discussion:-}

Orthopantomograms are one of the essential diagnostic aids used as a part of the investigations during orthodontic diagnosis and treatment planning. It is mainly used for the study of bone pattern, bone and root pathology, presence of supernumerary teeth, root shape and size, evaluation of TMJ, right and left facial symmetry, etc. Its main advantage lies in the evaluation of both maxillary and mandibular arches with Temporomandibular joint and their 
supporting structures. In addition to these benefits, it is highly advantageous if skeletal relationships can also be assessed using an orthopantomogram, thereby minimizing the requirement of a lateral cephalogram only for extensive skeletal assessments.

There are very few studies involving the use of panoramic radiographs in evaluating dentoskeletal specifications and they focus mainly on inter-condylar asymmetries and gonial angle measurements. Maxillary and mandibular measurements were taken independently, because the bite plate separates the maxillary and mandibular teeth during the panoramic exposure. Although the plates are necessary to eliminate the superimposition of corresponding teeth, they move the mandible forward and eliminate the overjet. Vertical linear measurements on the condyle and the ramus are not reliable for patients with macerated skulls. Also horizontal measurements were found to be unreliable.[11,12] Therefore only angular measurements were made on the panoramic Radiographs. One of the six keys of normal occlusion suggested by Andrews is the mesiodistal crown angulations. For occlusion to be considered normal the gingival part of the long axis of the crown must be distal to the occlusal part of the line. Different teeth exhibit different crown angulations. By measuring the angulations of each tooth in the study sample norms for mesiodistal inclinations and angulations can be calculated which would aid in judgment of treatment outcome.[8]

\section{Skeletal pattern - comparison of skeletal parameters between OPG and lateral Cephalogram (Table VI and IX):-}

The gonial angle (Co-Go/Go-Me) to OMAND (Co-Mc/Mc-Me) showed a Positive correlation, $\mathrm{r}=0.318$. This is in accordance with the study by KeijiMatia et al in which they evaluated the gonial angle (OMAND) from the OPG and the lateral cephalograms and determined that they could be determined with the same degree of accuracy. They also determined that the right and the left gonial angles can be quite easily determined individually from OPGs thus avoiding the disturbing influence of the superimposed images found on lateral cephalograms. Thus OPGs are a more obvious choice for determination of the gonial angles. [39] The palatal plane to mandibular plane angle (ANS$\mathrm{PNS} / \mathrm{Go}-\mathrm{Me}$ ) in the Lateral cephalogram showed a negative co-relation with OCOND (Co-Mc/ $\mathrm{Mc}-\mathrm{FMe})$ in the $\mathrm{OPG}, \mathrm{r}=0.279$, indicating that as the Palatal plane/ Mandibular plane increased there is a corresponding decrease in the OCOND. This is in accordance with the findings of Akcam et al [1]. This indicates that the palatal plane/ Mandibular plane can be predicted with a reasonable degree of accuracy using the OCOND from the orthopantomogram. The prediction value R2 was found to be $7.8 \%$.

\section{The mandibular plane angle (Go-Gn/S-N) to OCOND (Co-Mc/Mc-FMe):-}

In the OPG showed a negative correlation, $\mathrm{r}=-\mathrm{O}$.397. This was similar to the findings of the study by Akcam et al where the mandibular plane angle in the cephalograms increased with a corresponding decrease in the OCOND of the orthopantomogram. This decrease can be predicted with accuracy as shown by the prediction value R2 which was found to be $15.8 \%$.[1]

\section{The condylar inclination angle (Co-Go/Go-Me):-}

From the lateral cephalogram showed a positive correlation $r=0.318$ with OMAND (Co-Mc/Mc-Me) in the OPG. This is in accordance with the study conducted by Akcam et al in which they determined that the condylar inclination angle can be predicted with reasonable certainty from the OMAND of the OPG.[1]

In the present study a prediction value $\mathrm{R} 2$ of $10.1 \%$ was noted. The prediction value between the mandibular plane angle and OCOND is $15.8 \%$. Thus the level of prediction is high enough so that there is a possibility of predicting the cephalometric parameters from the panoramic measurements. This is in accordance with the study by Akcam et al. The prediction of the lateral cephalometric values from the OPG parameters was done by using the regression equations obtained through the statistical analysis.

Go $-\mathrm{Gn} / \mathrm{S}-\mathrm{N}=49.711( \pm 7.126)-0.46( \pm 0.139) *$ OCOND

ANS $-\mathrm{PNS} / \mathrm{Go}-\mathrm{Me}=34.278( \pm 5.829)-0.253( \pm 0.114) *$ OCOND

$\mathrm{Co}-\mathrm{Go} / \mathrm{Go}-\mathrm{Me}=72.864( \pm 19.425)+0.367( \pm 0.144) *$ OMAND 
GRAPH No. I:-Mean and standard deviations calculated for parameters measured from 60 OPGs

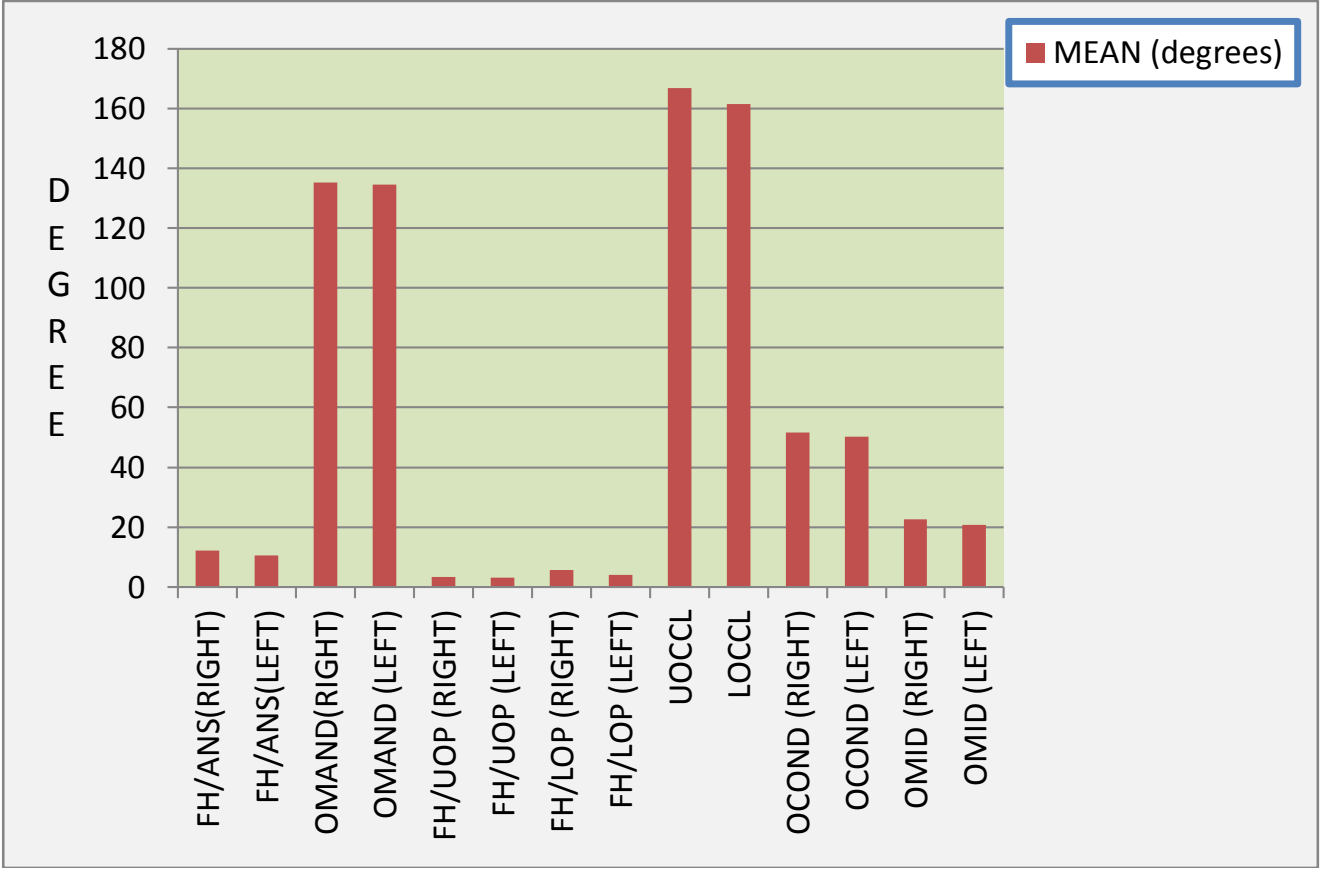

GRAPH No. II:-Comparison between males and females of the parameters measured from lateral cephalograms using students ' $t$ 'test.

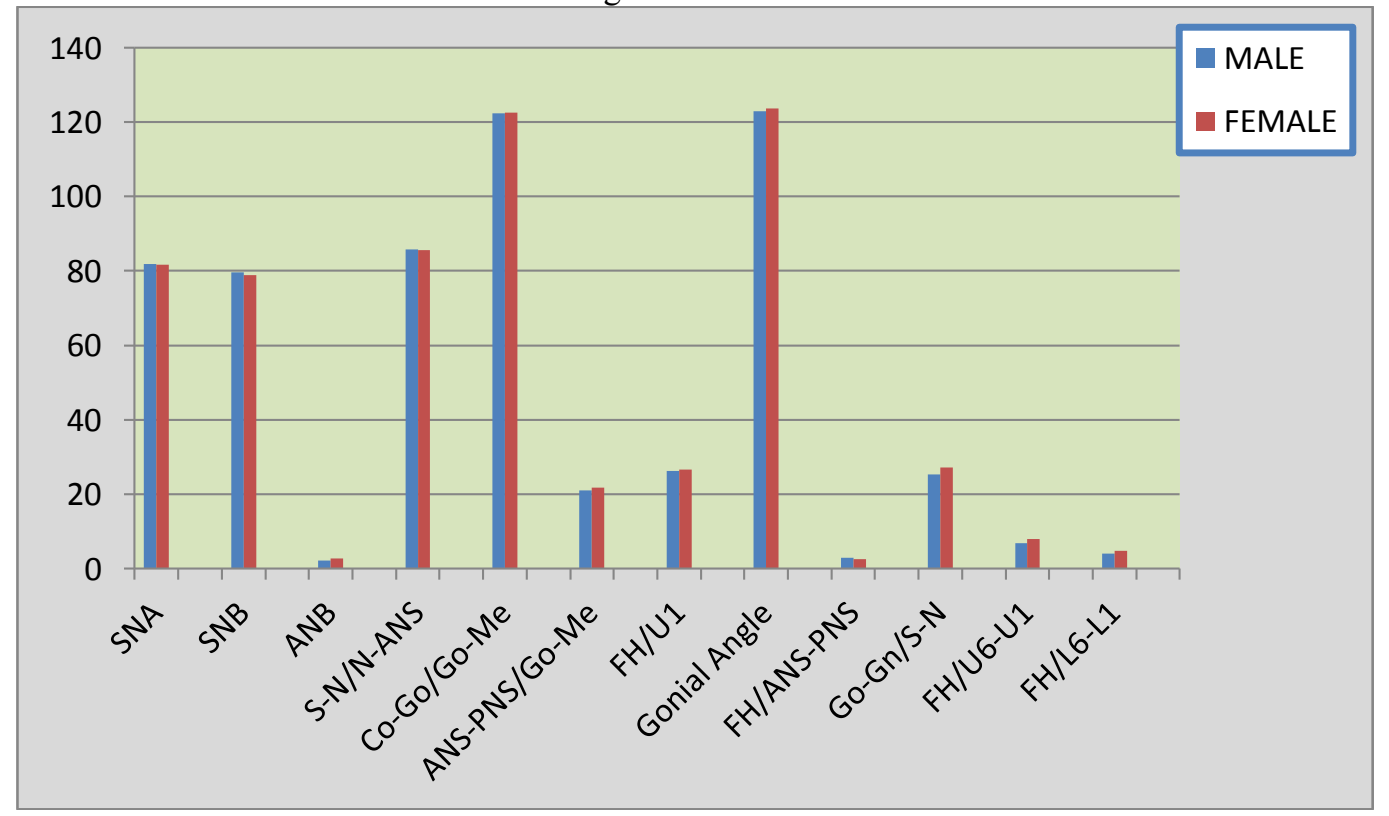


GRAPH No III:-Comparison between males and females of the parameters measured from panoramic radiographs using students ' $\mathrm{t}$ ' test.

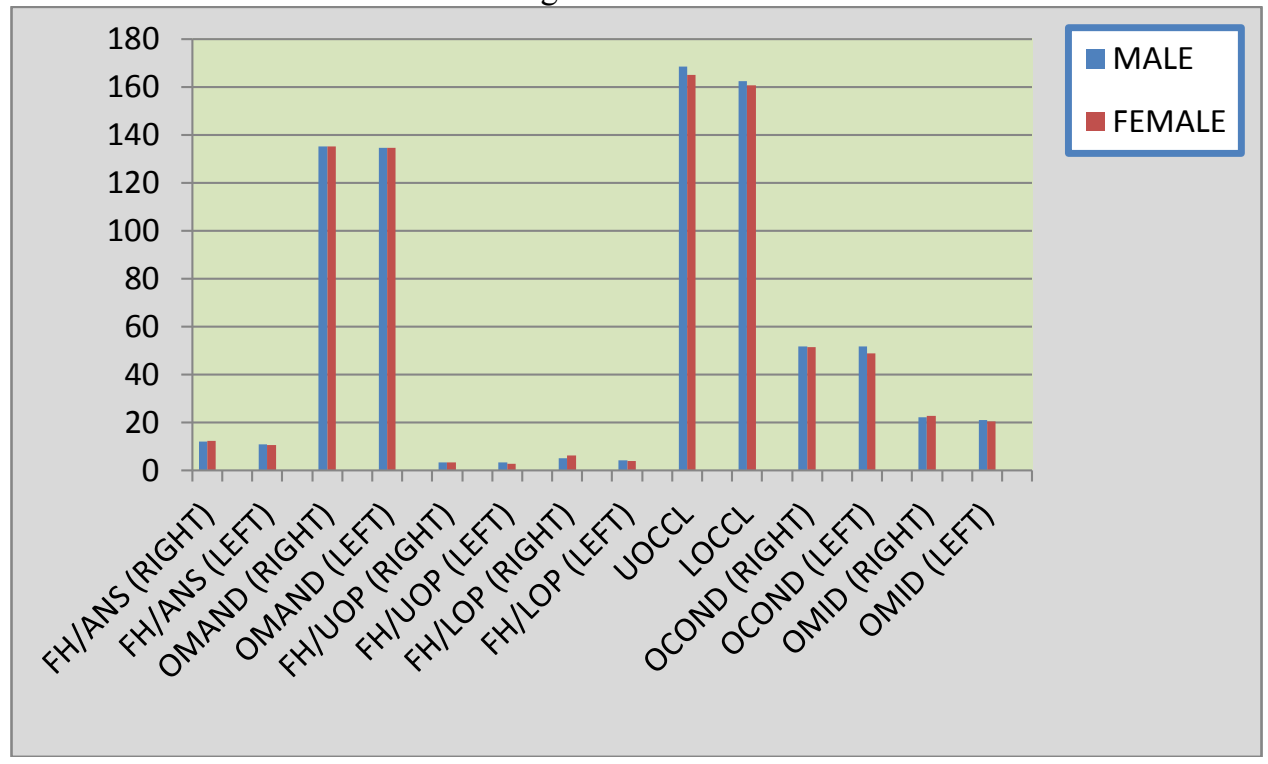

GRAPH IV:-Comparison between right and left sides of parameters measured from 60 panoramic radiographs using paired sample test.

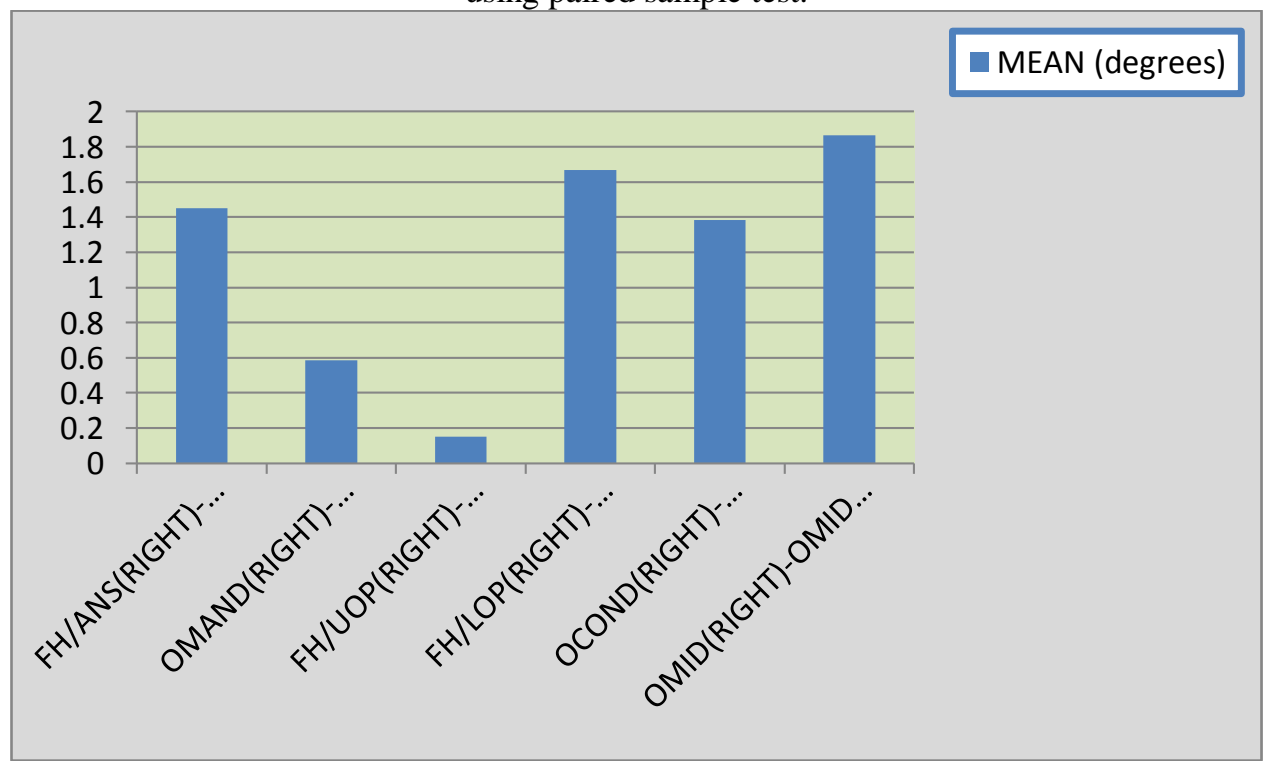


GRAPH V:-Correlation coefficient of dental parameter between OPG and Cephalometrics. Correlation coeficent of dental parametre between $O P$ Gand cephalometrics

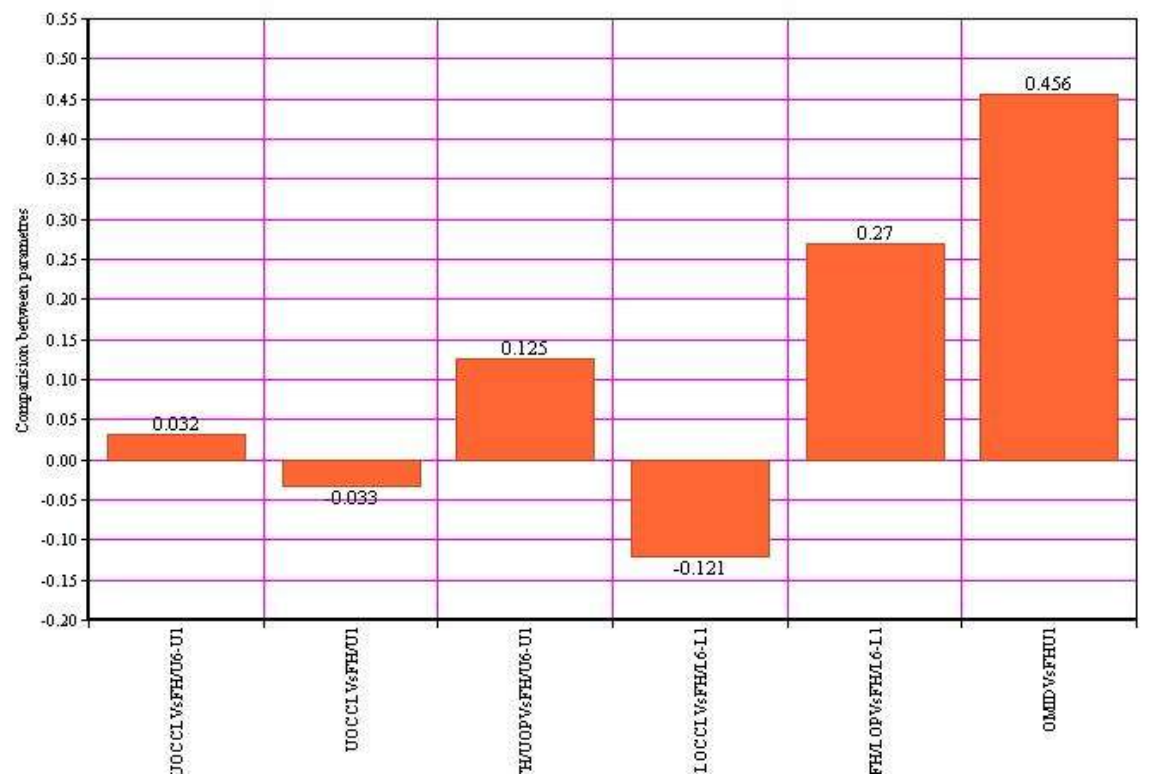

Tooth angulations and inclinations- comparison of dental parameters between OPG and lateralcephalogram (Table VII \& VIII):-

The UOCCL (U6-U1-U6, the angle formed by the molars of both sides with the incisors) of the OPG was correlated with the FH/U6-U1 (Frankfort's Horizontal plane to the plane joining upper first molar and central incisor) and the FH/UI (Frankfort's horizontal plane to the plane joining upper central Incisor) angles of the lateral cephalogram.

The $r$ values were found to be $\mathrm{r}=0.032$ for $\mathrm{UOCCL}$ with $\mathrm{FH} / \mathrm{U} 6-\mathrm{U} 1$

$r=-0.033$ for UOCCL with $\mathrm{FH} / \mathrm{U} 1-\mathrm{Po}$

This indicates a mild positive correlation between UOCCL and FH/U6-U1 and a mild negative correlation between UOOCL and FH/ U1. In this study the FH/UOP and FH/U6-U1 were positively correlated, $r=0.39$.

\section{LOCCL angle (L6-L1-L6):-}

In OPG was negatively correlated with FH/L6-LI (Frankfort's horizontal plane to the plane joining lower first to the lower central Incisor of the lateral cephalogram), $\mathrm{r}=-0.121$. In this study a significant positive correlation between FH/LOP and FH/L6 -L1, r = 0.270 was found. This correlation shows that, UOOCL, FH/ UOP, FH/LOP and OMID can be used to predict FH/ U1, FH/U6 - U1, FH/ L6-L1 respectively utilizing corresponding regression equation.

\section{Comparison of OPG parameters between right and left sides:-}

Skeletal parameter:-

Comparison of OMAND (panoramic alternative of gonial angle) and OCOND (panoramic alternative of condylar inclination angle) between the right and left sides in the OPG were statistically insignificant. A significant difference was found in the study by Akcam et all for the measurements obtained between the right and left sides of the OPG but no such difference was found in this study.[1 ]

\section{Dental parameters:-}

Comparison of FH/UOP (angle between maxillary occlusal planes) between the right and left sides in the orthopantomograms were statistically insignificant.

On completion of this study it can be noted that on comparison of the skeletal parameters between the OPG and cephalometric parameters such as the gonial angle to the OMAND (panoramic alternative), palatal plane: 
mandibular plane to the OCOND and mandibular plane angle to OCOND, all produced statistically significant values and with the help of the regression equations obtained. OPGs could be used to determine the skeletal relationship with a certain degree of predictability. On comparison of the dental parameters it was seen that the following panoramic parameters i.e. UOCCL (upper occlusal plane), FH/ UOP (angle between FH and maxillary occlusal planes), FH/ LOP (angle between FH and mandibular occlusal planes) and OMID (angle between FH and maxillary incisors) could be used to obtain the following cephalometric measurements i.e. FH/U1, FH/U6-U1 and FH/L6-L1.

On comparison of OPG parameters between the right and left sides, both Skeletal and dental parameters showed statistically insignificant differences which meant that measurements could be obtained from one side only.

In this study we also obtained the norms for mesiodistal axial inclinations and labio-lingual angulations by measuring these values for 28 teeth from all 60 OPGs and calculating the mean of all these values. This can aid us in the evaluation of final post treatment results. But in a study conducted by suitability of panoramic radiograph in assessing the mesiodistal angulation of teeth it was found that plane film techniques were more accurate than the panoramic technique.[41]

Therefore further investigation can be directed towards the practitioner's ability to discriminate different degrees of root angulation and parallelism should be contemplated along with evaluating similar parameters in different sample such as Class III malocclusions and class II div II malocclusions and determining the predictability percentages of the parameters discussed.

\section{Conclusions:-}

The array of investigative tools for the purpose of diagnosis and treatment planning is vast in the field of orthodontics. The most commonly used tools are the lateral cephalogram and OPG. While the lateral cephalogram is used for a quantitative description of the skeletal and dental status of an individual the OPG is an indispensable orthodontic screening tool in providing information about the teeth, their axial inclinations, maturation periods and surrounding tissues. This is mostly qualitative in nature. The OPG can be used in the quantitative assessment of a malocclusion but the predictability percentage has been found to be very low; therefore the clinician should be quite vigilant in this aspect. Angular measurements of the OPG can be used as a substitute for the corresponding angular measurements from the lateral cephalogram, both for dental and skeletal parameters with the help of regression equations. Either right or left side measurements in the OPG can be used for certain parameters instead of measuring both sides separately, as they are not significantly different. The OPG can be used to determine the norms for mesiodistal axial inclinations and labio-lingual angulations and these can further be used for evaluating treatment results on completion of orthodontic therapy. Standard exposure conditions and high image quality, panoramic radiograph can provide information on the vertical dimensions of dentofacial structures; however they are not reliable enough to give acceptably accurate additional information compared with lateral cephalograms. 